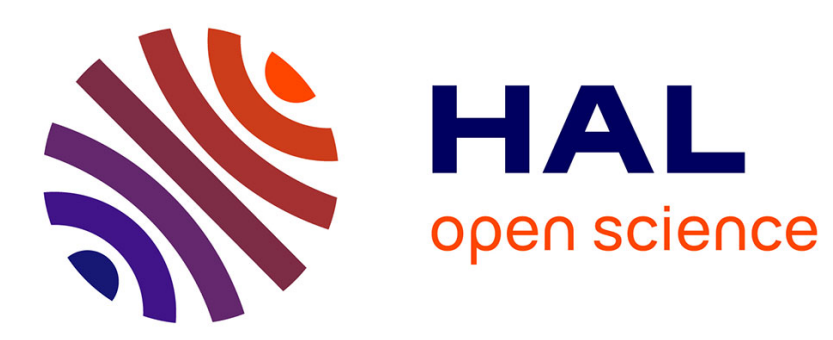

\title{
Identification of spider-mite species and their endosymbionts using multiplex PCR
}

Flore Zélé, Mylene Weill, Sara Magalhães

\section{To cite this version:}

Flore Zélé, Mylene Weill, Sara Magalhães. Identification of spider-mite species and their endosymbionts using multiplex PCR. Experimental and Applied Acarology, 2018, 74 (2), pp.123-138. 10.1007/s10493-018-0224-4 . hal-01943942

\section{HAL Id: hal-01943942 \\ https://hal.science/hal-01943942}

Submitted on 28 Oct 2020

HAL is a multi-disciplinary open access archive for the deposit and dissemination of scientific research documents, whether they are published or not. The documents may come from teaching and research institutions in France or abroad, or from public or private research centers.
L'archive ouverte pluridisciplinaire HAL, est destinée au dépôt et à la diffusion de documents scientifiques de niveau recherche, publiés ou non, émanant des établissements d'enseignement et de recherche français ou étrangers, des laboratoires publics ou privés. 


\section{Identification of spider-mite species and their endosymbionts using}

\section{2 multiplex PCR}

3 Flore Zélé ${ }^{*}$

$4 \quad$ Email: fezele@,fc.ul.pt

5 ORCID: 0000-0003-2954-5488

6 Mylène Weill ${ }^{2}$

7 Email: mylene.weill@umontpellier.fr

8 ORCID: 0000-0002-4043-1601

9 Sara Magalhães ${ }^{1}$

10 Email: snmagalhaes@fc.ul.pt

11 ORCID: 0000-0002-8609-7768

12

$13{ }^{1}$ Centre for Ecology, Evolution and Environmental Changes (cE3c), Faculdade de Ciências 14 da Universidade de Lisboa, Edificio C2, $3^{\circ}$ Piso Campo Grande, 1749016 Lisbon, Portugal

$15{ }^{2}$ Institut des Sciences de l'Evolution (CNRS-Université de Montpellier-IRD-EPHE), 34095

16 Montpellier, CEDEX 5, France

$17{ }^{*}$ Corresponding author

18 


\section{Abstract}

20 Spider mites of the genus Tetranychidae are severe crop pests. In the Mediterranean, a few 21 species coexist, but they are difficult to identify based on morphological characters. 22 Additionally, spider mites often harbour several species of endosymbiontic bacteria, which 23 may affect the biology of their hosts. Here, we propose novel, cost-effective, multiplex 24 diagnostic methods allowing a quick identification of spider-mite species as well as of the endosymbionts they carry. First, we developed, and successfully multiplexed in a single PCR, primers to identify Tetranychus urticae, Tetranychus evansi and Tetranychus ludeni, some of the most common Tetranychids found in southwest Europe. Moreover, we showed that this method allows detecting multiple species in a single pool, even at low frequencies (up to 1/100), and can be used on entire mites without DNA extraction. Second, we developed another set of primers to detect spider-mite endosymbionts, namely Wolbachia, Cardinium and Rickettsia in a multiplex PCR, along with a generalist spider-mite primer to control for potential failure of DNA amplification in each PCR. Overall, our method represents a simple, cost-effective and reliable method to identify spider-mite species and their symbionts in natural field populations, as well as to detect contaminations in laboratory rearings. This method may easily be extended to other species.

\section{$37 \quad$ Keywords}

Tetranychidae, Wolbachia, Cardinium, Rickettsia, species identification, multiplex diagnosis tool 
Spider mites (Tetranychidae) are ubiquitous herbivorous crop pests that occur on several plant species (Migeon and Dorkeld 2006-2017). In South-Western Europe, the most prevalent species found in natural populations are Tetranychus urticae, T. evansi and T. ludeni (Zélé et al. 2018). Whereas T. urticae is a generalist species, occurring on more than 900 host-plant species, T. ludeni is mainly found on plants in the order Solanales and T. evansi is a specialist of plants in the family Solanaceae (Migeon and Dorkeld 2006-2017). Thus, they often cooccur in the same field and even on the same individual plant (Ferragut et al. 2013). Given their minute size, phenotypic similarity, and scarcity of diagnose characters, the identification of these organisms at the species level is often a difficult task (Matsuda et al. 2013; Skoracka et al. 2015). To overcome this, several molecular methods, based on the diversity of the internal transcribed spacer region 2 of nuclear ribosomal DNA (ITS2), have been proposed to distinguish among spider-mite species and are currently under use (Ben-David et al. 2007; Hurtado et al. 2008; de Mendonça et al. 2011; Arimoto et al. 2013; Matsuda et al. 2013; Li et al. 2015; Shim et al. 2016). However, all methods described so far are relatively costly or time-consuming, as they require either sequencing the PCR products or using Restricted Fragment Length Polymorphisms (RFLP). The development of multiplex PCR, a reliable, cheap and quick method, to routinely identify arthropod species (e.g., Hosseini et al. 2007; Staudacher et al. 2011), including within the Acari (Perez-Sayas et al. 2015), appears to be an excellent solution to overcome these issues. Spider mites (and the Acari in general) are often colonized by several endosymbiotic bacteria, at high incidence relative to most other arthropod groups (Weinert et al. 2015). Indeed, herbivorous spider mites are commonly infected with Wolbachia and Cardinium (Gotoh et al. 2003; Liu et al. 2006; Gotoh et al. 2007b; Xie et al. 2011; Ros et al. 2012; 

al. 2018), and their phenotypic effects on spider mites are as yet unknown. In contrast, several studies have shown that Wolbachia and Cardinium have diverse fitness effects on their spider-mite hosts. In particular, they may manipulate the reproduction of their hosts via the induction of different degrees of cytoplasmic incompatibility (Vala et al. 2002; Gotoh et al. 2003; Gotoh et al. 2007a; Gotoh et al. 2007b; Ros and Breeuwer 2009; Xie et al. 2011; Zhu et al. 2012; Suh et al. 2015; Xie et al. 2016), a mechanism by which the cross between an infected male and an uninfected female results in embryonic mortality (Werren et al. 2008; Engelstadter and Hurst 2009; Ma et al. 2014). Apart from this common phenotypic effect, both bacteria can induce other types of reproductive manipulation such as feminization (i.e., genetic males developing as females) by Cardinium in the false spider-mite Brevipalpus phoenicis (Weeks et al. 2001), parthenogenesis induction (i.e., asexual daughter development) by Wolbachia in the genus Bryobia (Weeks and Breeuwer 2001), and hybrid breakdown by Wolbachia in T. urticae (Vala et al. 2000). Moreover, based on phenotypic characterisation and sequence comparisons (usually the Wolbachia wsp gene and the Cardinium 16S rDNA), multiple strains of these symbionts can be found in spider-mite populations (Gotoh et al. 2003; Liu et al. 2006; Gotoh et al. 2007a; Gotoh et al. 2007b; Yu et al. 2011; Ros et al. 2012; Zhang et al. 2016) and these two bacterial genera have been found infecting the same individual host (Ros et al. 2012; Zhu et al. 2012; Zhao et al. 2013a; Zhao et al. 2013b; Xie et al. 2016). In addition, Wolbachia and Cardinium can induce variable fitness effects in spider mites. For example, they can either decrease (PerrotMinnot et al. 2002; Ros and Breeuwer 2009; Suh et al. 2015), increase (Vala et al. 2002; Gotoh et al. 2007b; Xie et al. 2011; Zhao et al. 2013b), or not affect (Breeuwer 1997; Vala et 

the fecundity of their spider-mite hosts. These endosymbionts are thus likely to affect the distribution of spider mites and characterising their community in natural host populations is a timely issue. Although such symbionts can easily be identified using specific primers, attempts to develop a multiplex method to detect them are surprisingly scarce. Indeed, we found a report of two multiplex diagnostic methods allowing to identify up to seven symbionts in whiteflies: Portiera, Hemipteriphilus, Cardinium and Rickettsia on the one hand, and Portiera, Hamiltonella, Arsenophonus and Wolbachia on the other (Kurata et al. 2016); and another study allowing to identify Wolbachia and Cardinium in spider mites (Chen et al. 2009). However, to our knowledge, a multiplex allowing the simultaneous detections of Wolbachia, Cardinium and Rickettsia has to date not been developed. Moreover, none of these studies have performed the simultaneous amplification of host DNA, which allows the exclusion of false negatives for each single PCR. Here we present a simple, cost-effective and reliable method to identify spider-mite species and their symbiotic community, focussing on the mite species we find in the Iberian 105 Peninsula. This method can be applied to characterise field communities as well as to detect contaminations in laboratory populations, and may be extended to other species in the future.

\section{Materials and Methods}

\section{Spider-mite populations}

110 A total of 22 spider-mite populations, belonging to three different species (T. urticae green 111 and red forms, T. ludeni and T. evansi), were used in this study. Some of these populations 112 were collected by our group in 2013 (Zélé et al. 2018), others were kindly provided by A. 113 Janssen and by T. Van Leeuwen (see Electronic supplementary materials, Table S1, for a full 114 description of these populations). The populations were maintained in our laboratory under 
115 standard conditions $\left(25 \pm 2{ }^{\circ} \mathrm{C}, 60 \% \mathrm{RH}, 16 / 8 \mathrm{~h} \mathrm{~L} / \mathrm{D}\right.$; Clemente et al. 2016) in mass-rearing

116 insect-proof cages containing either bean cv. Contender seedlings (obtained from Germisem,

117 Oliveira do Hospital, Portugal) for T. urticae and T. ludeni, or tomato cv. Money Maker 118 seedlings (obtained from Mr. Fothergill's Seeds, Kentford, UK) for T. evansi. The species and 119 infection status of each population was known prior to this experiment (based on earlier 120 sequencing of the wsp gene of Wolbachia, of the 16S rDNA of Cardinium, of the gtlA gene of 121 Rickettsia, and of complete sequences of spider-mite rDNA region including ITS1, 5.8S 122 rRNA, ITS2 and partial fragments of 18S and 28S rRNA from individuals and pools of mites 123 from each population following at least one year of laboratory rearing; data not shown). Some 124 of the populations infected by Wolbachia and/or Rickettsia were treated with tetracycline 125 hydrochloride $(0.1 \%, \mathrm{w} / \mathrm{v})$ for three successive generations (Breeuwer 1997) to obtain their 126 uninfected counterparts (the suffix ".tet" has been added to the name of the population in this 127 case). During the treatment, mites were maintained in petri dishes containing bean (or tomato) 128 leaf fragments placed on cotton wet with the solution. At each generation 50 adult mated 129 daughters were transferred to a new petri dish containing fresh leaf fragments and solution. At 130 the third generation after treatment, 14 individual females and a pool of 100 females were 131 taken from each colony and checked by PCR to confirm that they were uninfected. These 132 colonies were maintained in a mass-rearing environment without antibiotics for about five 133 generations before this study, to avoid (or limit) potential side effects of antibiotic treatment 134 (Ballard and Melvin 2007; Zeh et al. 2012; O'Shea and Singh 2015).

\section{Primer design and multiplex PCR for spider-mite identification}

137 We developed a multiplex PCR to identify the three spider-mite species commonly found in 138 the field samples that we have collected in South-Western Europe, namely T. urticae, T. 139 ludeni and T. evansi (Zélé et al. 2018). A Tetranychus-generalist forward primer and three 
140 different species-specific reverse primers (Table 1) were designed manually using an 141 alignment of 38 complete Tetranychus sequences of the rDNA region including ITS1, 5.8S 142 rRNA, ITS2 and partial fragments of 18S and 28S rRNA from GenBank and belonging to 18 143 species (Supplementary Table S2). Across several sets of primers designed to meet the criteria 144 of a multiplex PCR (Henegariu et al. 1997; Markoulatos et al. 2002; Sint et al. 2012), the best

145 set of spider-mite generalist primers obtained allow the amplification of partial 5.8S rDNA, 146 ITS2 and 28S rDNA gene sequences (Fig. 1). Primer concentrations in the mix (Table 1) were 147 empirically adjusted to ensure an even amplification of all targeted DNA fragments (Sint et 148 al. 2012). PCRs were performed using QIAGEN Multiplex PCR materials (Qiagen NV, 149 Venlo, The Netherlands) and optimized for PCR using DNA extracts in a $10 \mu 1$ final reaction 150 volume (Table 2). Total genomic DNA was extracted either from individual or pooled mites 151 using the Sigma-Aldrich protocol (GenElute $^{\mathrm{TM}}$ Mammalian Genomic DNA Miniprep Kit, 152 Sigma-Aldrich, St. Louis, MO, United States). Amplification conditions were as follows: $15315 \mathrm{~min}$ at $95^{\circ} \mathrm{C}$, followed by 35 cycles of $94^{\circ} \mathrm{C}$ for $30 \mathrm{~s}, 58^{\circ} \mathrm{C}$ for $1 \mathrm{~min} 30 \mathrm{~s}, 72^{\circ} \mathrm{C}$ for $1 \mathrm{~min}$ and 154 a final step at $72^{\circ} \mathrm{C}$ for $10 \mathrm{~min}$. Following the PCR, $5 \mu \mathrm{L}$ of each PCR product was 155 electrophoresed on a $\%$ agarose gel, stained with Envision ${ }^{\mathrm{TM}}$ DNA Dye as Loading Buffer 156 (Amresco Inc., Solon, USA), and photographed under ultra-violet light.

Primer design and multiplex PCR to detect simultaneously Wolbachia, Cardinium and

\section{Rickettsia}

160 To detect infection by Wolbachia, Cardinium and Rickettsia, bacterial-specific primers (Table 161 3) were designed using PrimerPlex (Multiplex PCR Primer Design Software; PREMIER 162 Biosoft International, Palo Alto, USA), on the basis of the wsp gene of Wolbachia, of the 16S 163 rDNA of Cardinium, and of the gtlA gene of Rickettsia, along with spider-mite generalist 164 primers (to control the DNA quality and discriminate uninfected individuals from PCR 
165 failure) on the basis of complete Tetranychus sequences of the rDNA region including ITS1, 166 5.8S rRNA, ITS2 and partial fragments of 18S and 28S rRNA gene as above. After designing 167 and testing several primers sets, the best obtained locates in the ITS1 region (Table 3). DNA 168 extraction, PCRs (Table 2), amplification conditions (with an annealing temperature of $65^{\circ} \mathrm{C}$ ), 169 and gel electrophoresis, were performed as previously described for species identification.

170 Tests were done in T. urticae, as this was the only mite species harbouring endosymbiont in 171 our laboratory populations at the time of this study. Template DNAs came from pools of $T$. 172 urticae females known to be uninfected, infected by Wolbachia only, by Cardinium only, or 173 co-infected with Wolbachia and Rickettsia (we were unable to find mites infected with the 174 latter only). To obtain other amplification profiles (e.g., Wolbachia-Cardinium co-infection or 175 triple infection; Fig. 3), equal volumes of different DNA extracts were mixed prior to perform 176 the PCR.

\section{Sensitivity tests}

179 We tested the sensitivity of both multiplex PCRs on DNA extracts from different sizes of 180 female pools $(10,25,50$ and 100 individuals) in which only one female from a different species or with a different infection status (i.e., by Wolbachia, Rickettsia, or both) was added. We then checked whether two profiles, one corresponding to the original pool, the other to the 183 introduced female, could be detected.

\section{Application to non-DNA extracted individuals}

186 We tested the efficiency of the multiplex PCRs for endosymbiont detection at the individual 187 level on non-DNA extracted adult females and males. Here, entire mites were placed directly 188 in the PCR tube, subsequently frozen at $-20^{\circ} \mathrm{C}$ during 15 minutes then briefly centrifuged 189 before adding the PCR mix. The PCR mix were optimized for using entire mites (Table 2), 
while amplification conditions and gel electrophoresis were performed as previously

191 described for PCR using DNA extracts. We tested 4 adult T. urticae females and males that 192 were either uninfected, infected with Wolbachia only, or coinfected by Rickettsia and 193 Wolbachia.

\section{Application to detect contamination in spider-mite laboratory populations}

We applied both multiplex PCRs to detect potential contaminations by spider-mite individuals

(i.e., from a different species and/or with a different infection status) across 29 different mite populations in the laboratory. From each population, we created a pool of 100 females and extracted DNA as previously described. For each pool we performed both "species 200 identification" and "endosymbiont detection" multiplex PCRs.

\section{Results}

\section{Multiplex PCR to detect spider-mite species and symbionts}

204 Overall, our method allowed the unambiguous identification of the three target spider-mite 205 species of this study: T. urticae, T. ludeni and T. evansi (Fig. 2), and of the three symbiont 206 species found in T. urticae (Fig. 3). Further, using several sequences from genebank 207 (Supplementary Table S2), we could establish that the generalist primer designed in this study 208 (TspG_F) should anneal with the partial 5.8S rDNA sequences from 18 different Tetranychus 209 species. However, while both T. ludeni-specific primer (TIS_R) and T. evansi-specific primer 210 (TeS_R) are highly specific to these two species (i.e., they should bind only to the ITS2 211 sequences from T. ludeni, and T. evansi, respectively), the T. urticae-specific primer (TuS_R) 212 might bind to the 28S rDNA gene sequences of few other species, but not to that of many 213 others, including T. evansi and T. ludeni (Supplementary Table S2). At the annealing 214 temperature used in our protocol $\left(\mathrm{Tm}=65^{\circ} \mathrm{C}\right)$, the Tetranychus-generalist primers (ITS2G_F 
and ITS2G_R), designed for the "endosymbiont detection" multiplex PCR, should bind to the

216 ITS1 sequences of several Tetranychus species (i.e., despite the occurrence of some SNPs

217 between the primers sequence and the target site of these primers due to the high intraspecific

218 variability of the ITS1 region, which makes it unsuitable for species discrimination, Navajas

219 et al. 1999; Hurtado et al. 2008), and the bacterial-specific primers blast to an extensive

220 number of sequences of their respective target symbionts obtained from many different

221 arthropod host species, including T. urticae and several other spider-mite species (Primer-

222 Blast results; data not shown).

223

224 Sensitivity tests

225 For species identification (Fig. 4) and endosymbiont detection (Fig. 5), we could detect 226 contaminations by a single mite for all sizes of pools, although the strength of the signal 227 decreases with increasing pool size in the multiplex PCR for species identification (i.e. the 228 relative abundance of contaminant DNA decreases with increasing pool size: 1/10, 1/25, 1/50 229 and $1 / 100)$.

\section{Application to non-DNA extracted individuals}

232 We obtained 100\% success at detecting single infections by Wolbachia and coinfections 233 Wolbachia-Rickettsia, and no unspecific amplifications in uninfected individuals for both 234 females (Fig. 6a) and males (Fig. 6b).

236 Application to detect contamination in spider-mite laboratory populations

237 Amplifications of DNA from pooled individuals form laboratory populations was successful, 238 and, for 27 of 29 of them, the obtained profiles were those expected based on the known 239 species and endosymbiont composition (Fig. 7). However, for the two remaining populations 
240 ("Tl CVM" and "Tu FR"), which were expected to be symbiont-free based on earlier tests (cf.

241 Methods), we detected infections by Wolbachia (Fig. 7a). In the PCR profile for species

242 identification (Fig. 7b), the population "Tl CVM" showed a double amplification, for $T$.

243 ludeni and for T. urticae, while "Tu FR" showed only one band for T. urticae. The

244 combination of both results suggests that both populations have been contaminated by

245 Wolbachia-infected T. urticae mites from another population (note that this result matches

246 expectations since only T. urticae populations were infected with Wolbachia in our laboratory

247 at the time of this study). Although these contaminations might have occurred during DNA

248 extraction, or during PCR, subsequent tests (new DNA extractions followed by a standard

249 PCR of the Wolbachia wsp gene; data not shown) confirmed that the source of both

250 contaminations was Wolbachia-infected T. urticae mites during laboratory rearing.

\section{Discussion}

253 This study reports the development of multiplex PCR methods for rapid identification of $T$.

254 urticae, T. evansi and T. ludeni (Fig. 2), and detection of their endosymbionts, namely 255 Wolbachia, Cardinium and Rickettsia (Fig. 3). Given the difficulties in relying on 256 morphological characters, the identification of spider mites at the species level is often a 257 difficult task (Matsuda et al. 2013; Skoracka et al. 2015). Moreover, the efficient detection of 258 these endosymbionts, which often cause reproductive abnormalities in their host arthropods 259 (Werren et al. 2008; Engelstadter and Hurst 2009; Ma et al. 2014) provides important 260 information concerning the populations sampled. In mass diagnosis, the introduction of 261 multiplex PCRs reduces considerably the need of DNA template when DNA is limited or 262 precious, and the cost of labor time and reagents. Consequently, multiplex PCR systems are 263 increasingly used in biological studies as they allow simultaneous amplification of several

264 DNA fragments within one reaction.

This is a post-peer-review, precopyedit version of an article published in Experimental and 11 Applied Acarology. The final authenticated version is available online at: https://doi.org/10.1007/s10493-018-0224-4 
In multiplex PCR, short amplicon lengths and high annealing temperatures are crucial

266 for enhanced detection efficiency. Competition between different primers, due to differences

267 in GC content, degree of mismatch between primer and priming site, secondary structure or

268 interference with each other, can also influence the sensitivity and efficiency of amplification

269 of the target molecules (Henegariu et al. 1997; Markoulatos et al. 2002; Sint et al. 2012). The

270 primers presented in this study were designed to overcome these problems, to have high

271 melting temperatures (Tm), and produce DNA fragments of distinct, but short size, allowing

272 easy discrimination of the three spider mites species and of their endosymbiont by agarose gel

273 electrophoresis. In addition, the successful multiplex PCR amplification of the diagnostic

274 products is also dependent on the ratio of the primer pairs and the extending time (Sint et al.

275 2012). Series of optimization have thus been conducted to provide a protocol that improves

276 the detection success and ensures an even amplification of all targeted DNA fragments. These

277 optimizations, however, have been conducted using Qiagen materials, and we cannot ensure

278 the same results using different materials without performing novel series of optimizations.

Besides being time- and cost-effective, one of the main advantages of the multiplex

280 PCRs developed in this study concerns its utilisation on DNA extracted from pooled spider

281 mites. The sensitivity tests performed in this study shows the technique to be highly sensitive

282 and allows detecting up to one individual mite in a pool of 100 mites belonging to a different

283 species, or having a different infection status (Fig. 4 and Fig. 5). This can be particularly

284 useful, for instance, when founding new laboratory populations from field-collected

285 individuals, and to regularly test for contaminations between laboratory populations

286 belonging to different species or carrying different endosymbionts. Both multiplex PCRs

287 presented in this study are already routinely used in our own laboratory since their initial

288 development in 2014: pools of 100 adult females are collected from each of our populations

289 every 6 months, their DNA extracted and both multiplex PCRs systematically performed on

This is a post-peer-review, precopyedit version of an article published in Experimental and 
each sample (Fig. 7). This procedure is also carried out on each population before its use in any experiment performed.

Another important feature of these multiplex PCRs is the possibility to use them, at the individual level, on non-extracted spider mites for both laboratory and field studies. Indeed, they are easily applicable, for instance, to estimate species prevalence while studying interspecific competition or performing an invasion experiment (e.g., Sarmento et al. 2011; Sato et al. 2014; Orsucci et al. 2017), or to determine endosymbiont establishment and invasion in laboratory populations (e.g., $\mathrm{Xi}$ et al. 2005). Moreover, the use of these multiplex PCR on non-extracted single individuals can be particularly useful for quick and costeffective prevalence assessment on field-collected populations. Indeed, the multiplex PCR developed in this study to detect endosymbionts presents the main advantage, of including an internal control for failed PCR at the individual level (i.e., the inclusion of primers for host DNA amplification; Fig. 3). However, very low within-host densities of the target symbionts in field-collected mites might be difficult to detect using multiplex PCR without prior DNA extraction. In particular, we optimized the PCR mix to increase the specificity of amplification by increasing the volume of Q-solution (QIAGEN Multiplex PCR materials; Qiagen NV, Venlo, The Netherlands), but this can also result in a decreased sensitivity of the PCR. However, problems at detecting very low within-host densities of various parasite species also hold true for standard PCR methods (Gomez-Diaz et al. 2010; Lachish et al. 2012; Zélé et al. 2014). The sensitivity tests performed here, nevertheless, proved the technique to be highly sensitive and allows detection of a very low density of symbionts in pools of mites (Fig. 5), and both non-extracted individual females and males were successfully tested (Fig. 6), which confirmed the feasibility and reliability of this method.

A major issue with the application of these methods to detect endosymbionts is the use of a single primer pairs. Despite the fact that each pair of primers, to detect Wolbachia, 
315 Cardinium and Rickettsia, is generalist and blasts to an extensive number of sequences of 316 their respective target symbionts, past studies have revealed that using a combination of

317 primer pairs might be necessary to detect novel or rare symbiont strains (Simões et al. 2011).

318 The method developed here is thus particularly useful for the detection of expected 319 symbionts, but does not guarantee detection of novel strains. In line with this, standard PCR 320 methods and sequencing should be used following the detection of symbionts in newly 321 collected field populations to confirm the infection and detect potential false positives for 322 these bacteria (Duron et al. 2008). While using non-extracted DNA, another issue is the 323 impossibility to perform several tests on each individual (the entire body is placed in the PCR 324 mix). This can be particularly problematic when having a limited number of individuals. In 325 this case, we would rather advise to perform a standard DNA extraction prior to the multiplex 326 PCR. Another solution, however, to determine the species and endosymbiont infection from a 327 single female individual, would be to allow a female to oviposit in the laboratory before 328 performing the multiplex PCR for endosymbiont detection without DNA extraction, and to 329 keep its offspring, to determine a posteriori its species by performing the multiplex PCR for 330 species determination on the offspring.

331 Here, we developed a multiplex PCR to detect, and discriminate between, T. urticae, 332 T. evansi and T. ludeni. We made this choice because these are the species that were present 333 in the samples we have been collecting in the field in the Iberian Peninsula and Southern 334 France (Zélé et al. 2018). Using several sequences from GenBank, we could establish that our 335 reverse primers allowing the identification of $T$. evansi and $T$. ludeni are highly specific, but 336 the one allowing the identification of T. urticae may also allow the detection of other spider 337 mites species (Supplementary Table S2), thus hampering their discrimination. In the case of 338 Southern Europe, the main concern is T. turkestani, which has been found in both Spain and 339 Portugal (Migeon and Dorkeld 2006-2017). However, we have failed to find this species in 
340 the several field surveys performed in the Peninsula since 2009. In any case, this multiplex

341 PCR should be extended for the detection and discrimination of many other spider-mite

342 species. For instance, multiplex PCR assays have also been developed to rapidly identify,

343 within a single-step reaction, nine different species of the click beetle larvae within the genus

344 Agriotes (Staudacher et al. 2011). In particular, the suitability of the species-specific primers

345 developed by Shim et al. (2016), for T. urticae, T. kanzawai, T. phaselus and T. truncates for

346 multiplex PCR could be tested, as they produce different size product and have relatively

347 similar melting temperature. Similarly, the multiplex PCR developed here for endosymbiont

348 detection could be extended to detect other endosymbionts such as Spiroplasma, that also

349 occasionally infects spider mites (Zhang et al. 2016; Staudacher et al. 2017), and the host

350 generalist primers could be designed in more conserved regions such as the 18S (Monzó et

351 al. 2010). For instance, Kurata et al. (2016) developed a multiplex PCR to identify up to

352 seven endosymbionts species in whiteflies (within two different multiplex PCR assays) but it

353 does not allow the detection of Spiroplasma.

354 Finally, the use of multiplex PCRs without DNA extraction could be adapted to other

355 arthropod species, since non-extracted DNA from first instar larvae of some insect species are

356 already being used in simplex PCR (e.g., for the mosquito Culex pipiens, Milesi et al. 2017);

357 as well as in non-arthropods samples, as shown for non-extracted genital swabs for HSV

358 diagnostic (Pandori et al. 2006; Miari et al. 2015).

359 In conclusion, the two multiplex PCR methods developed here for the identification of

360 T. urticae, T. evansi and T. ludeni, and for the detection of Wolbachia, Cardinium and

361 Rickettsia in spider mites, respectively, are rapid, reliable, specific, sensitive and cost-

362 effective. The assays are versatile since they can also be used directly on non-extracted spider

363 mites individuals. Consequently, this method is suitable for a wide variety of applications for 364 reliable species identification and assessment of infection status. Although this study focused

This is a post-peer-review, precopyedit version of an article published in Experimental and 15 Applied Acarology. The final authenticated version is available online at: https://doi.org/10.1007/s10493-018-0224-4 
365 only on three spider-mite species and on the detection of three endosymbionts in various

366 spider-mite species, these multiplex PCR methods could be widely developed for the

367 detection of several host species and arthropods endosymbionts, by redesigning sequence-

368 specific primers.

369

\section{Acknowledgments}

371 We are grateful to Olivier Duron for useful discussions and comments, and to Inês Santos for 372 taking care of plants and mite populations. This work was funded by an FCT-ANR project 373 (FCT-ANR//BIA-EVF/0013/2012) to SM and Isabelle Olivieri, and by an FCT-Tubitak 374 project (FCT-TUBITAK/0001/2014) to SM and Ibrahim Cakmak. FZ was funded through an 375 FCT Post-Doc fellowship (SFRH/BPD/125020/2016). Funding agencies did not participate in 376 the design or analysis of experiments. We declare that we do not have any conflict of interest.

\section{References}

Arimoto, M., M. Satoh, R. Uesugi, M. Osakabe (2013) PCR-RFLP analysis for identification of Tetranychus spider mite species (Acari: Tetranychidae). J Econ Entomol 106, 661668 doi: $10.1603 /$ ec12440

Ballard, J.W.O., R.G. Melvin (2007) Tetracycline treatment influences mitochondrial metabolism and mtDNA density two generations after treatment in Drosophila. Insect Mol Biol 16:799-802

Ben-David, T., S. Melamed, U. Gerson, S. Morin (2007) ITS2 sequences as barcodes for identifying and analyzing spider mites (Acari : Tetranychidae). Exp Appl Acarol 41, 169-181 doi:10.1007/s10493-007-9058-1 
Breeuwer, J.A.J. (1997) Wolbachia and cytoplasmic incompatibility in the spider mites Tetranychus urticae and T-turkestani. Heredity 79, 41-47 doi:10.1038/hdy.1997.121

Chen, X.L., R.R. Xie, G.Q. Li, X.Y. Hong (2009) Simultaneous detection of endosymbionts Wolbachia and Cardinium in spider mites (Acari: Tetranychidae) by multiplex-PCR. Int J Acarol 35, 397-403 doi:10.1080/01647950903292756

Clemente, S.H., L.R. Rodrigues, R. Ponce, S.A.M. Varela, S. Magalhães (2016) Incomplete species recognition entails few costs in spider mites, despite first-male precedence. Behav Ecol Sociobiol 70, 1161-1170 doi:10.1007/s00265-016-2124-0

de Mendonça, R.S., D. Navia, I.R. Diniz, P. Auger, M. Navajas (2011) A critical review on some closely related species of Tetranychus sensu stricto (Acari: Tetranychidae) in the public DNA sequences databases. Exp Appl Acarol 55, 1-23 doi:10.1007/s10493-0119453-5

Duron, O., D. Bouchon, S. Boutin, L. Bellamy, L.Q. Zhou, J. Engelstadter, G.D. Hurst (2008) The diversity of reproductive parasites among arthropods: Wolbachia do not walk alone. BMC Biol 6, 27 doi:10.1186/1741-7007-6-27

Engelstadter, J., G.D.D. Hurst (2009) The ecology and evolution of microbes that manipulate host reproduction. Annu Rev Ecol Evol Syst 40, 127-149 doi:10.1146/annurev.ecolsys.110308.120206

Ferragut, F., E. Garzon-Luque, A. Pekas (2013) The invasive spider mite Tetranychus evansi (Acari: Tetranychidae) alters community composition and host-plant use of native relatives. Exp Appl Acarol 60, 321-341 doi:10.1007/s10493-012-9645-7

Gomez-Diaz, E., P.F. Doherty, D. Duneau, K.D. McCoy (2010) Cryptic vector divergence masks vector-specific patterns of infection: an example from the marine cycle of Lyme borreliosis. Evol Appl 3, 391-401 doi:10.1111/j.1752-4571.2010.00127.x 
412 Gotoh, T., H. Noda, X.Y. Hong (2003) Wolbachia distribution and cytoplasmic incompatibility based on a survey of 42 spider mite species (Acari : Tetranychidae) in Japan. Heredity 91, 208-216 doi:10.1038/sj.hdy.6800329

Gotoh, T., H. Noda, S. Ito (2007a) Cardinium symbionts cause cytoplasmic incompatibility in spider mites. Heredity 98, 13-20 doi:10.1038/sj.hdy.6800881

Gotoh, T., J. Sugasawa, H. Noda, Y. Kitashima (2007b) Wolbachia-induced cytoplasmic incompatibility in Japanese populations of Tetranychus urticae (Acari : Tetranychidae). Exp Appl Acarol 42, 1-16 doi:10.1007/s10493-007-9072-3

Henegariu, O., N.A. Heerema, S.R. Dlouhy, G.H. Vance, P.H. Vogt (1997) Multiplex PCR: Critical parameters and step-by-step protocol. Biotechniques 23, 504-511

Hosseini, R., M.A. Keller, O. Schmidt, V.W. Framenau (2007) Molecular identification of wolf spiders (Araneae : Lycosidae) by multiplex polymerase chain reaction. Biol Control 40, 128-135 doi:10.1016/j.biocontrol.2006.10.007

Hoy, M.A., A. Jeyaprakash (2005) Microbial diversity in the predatory mite Metaseiulus occidentalis (Acari : Phytoseiidae) and its prey, Tetranychus urticae (Acari : Tetranychidae). Biol Control 32, 427-441 doi:10.1016/j.biocontrol.2004.12.012

Hurtado, M.A., T. Ansaloni, S. Cros-Arteil, J.A. Jacas, M. Navajas (2008) Sequence analysis

Kurata, A., A. Fujiwara, N. Haruyama, T. Tsuchida (2016) Multiplex PCR method for rapid identification of genetic group and symbiont infection status in Bemisia tabaci (Hemiptera: Aleyrodidae). Appl Entomol Zool 51, 167-172 doi:10.1007/s13355-015$0378-\mathrm{z}$ 
436 Lachish, S., A.M. Gopalaswamy, S.C.L. Knowles, B.C. Sheldon (2012) Site-occupancy modelling as a novel framework for assessing test sensitivity and estimating wildlife disease prevalence from imperfect diagnostic tests. Methods Ecol Evol 3, 339-348 doi:10.1111/j.2041-210X.2011.00156.X

Li, D.M., Q.H. Fan, D.W. Waite, D. Gunawardana, S. George, L. Kumarasinghe (2015) Development and validation of a real-time PCR assay for rapid detection of twospotted spider mite, Tetranychus urticae (Acari: Tetranychidae). Plos One 10, e0131887 doi:10.1371/journal.pone.0131887

Liu, Y., H. Miao, X.Y. Hong (2006) Distribution of the endosymbiotic bacterium Cardinium in Chinese populations of the carmine spider mite Tetranychus cinnabarinus (Acari : Tetranychidae). J Appl Entomol 130, 523-529 doi:10.1111/j.1439-0418.2006.01112.x

Ma, W.J., F. Vavre, L.W. Beukeboom (2014) Manipulation of arthropod sex determination by endosymbionts: diversity and molecular mechanisms. Sex Dev 8, 59-73

Markoulatos, P., N. Siafakas, M. Moncany (2002) Multiplex polymerase chain reaction: A practical approach. J Clin Lab Anal 16, 47-51 doi:10.1002/jcla.2058

452 Matsuda, T., C. Fukumoto, N. Hinomoto, T. Gotoh (2013) DNA-based identification of spider mites: molecular evidence for cryptic species of the genus Tetranychus (Acari: Tetranychidae). J Econ Entomol 106, 463-472 doi:10.1603/ec12328

Miari, V.F., G.R. Wall, D.A. Clark (2015) Evaluation of non-extracted genital swabs for realtime HSV PCR. J Med Virol 87, 125-129 doi:10.1002/jmv.23967

457 Migeon, A., F. Dorkeld (2006-2017) Spider Mites Web: a comprehensive database for the Tetranychidae. In. http://www.montpellier.inra.fr/CBGP/spmweb/ 
Milesi, P., M. Weill, T. Lenormand, P. Labbe (2017) Heterogeneous gene duplications can be adaptive because they permanently associate overdominant alleles. Evol Lett 1, 169180 doi: $10.1002 /$ evl3.17

Monzó, C., B. Sabater-Munoz, A. Urbaneja, P. Castanera (2010) Tracking medfly predation by the wolf spider, Pardosa cribata Simon, in citrus orchards using PCR-based gutcontent analysis. Bull Entomol Res 100, 145-152 doi:10.1017/s0007485309006920

Navajas, M., J. Lagnel, G. Fauvel, G. De Moraes (1999) Sequence variation of ribosomal internal transcribed spacers (ITS) in commercially important phytoseiidae mites. Exp Appl Acarol 23, 851-859 doi:10.1023/a:1006251220052

O'Shea, K.L., N.D. Singh (2015) Tetracycline-exposed Drosophila melanogaster males produce fewer offspring but a relative excess of sons. Ecol Evol 5, 3130-3139 doi:10.1002/ece3.1535

Orsucci, M., M. Navajas, S. Fellous (2017) Genotype-specific interactions between parasitic arthropods. Heredity 118, 260-265 doi:10.1038/hdy.2016.90

Pandori, M.W., J. Lei, E.H. Wong, J. Klausner, S. Liska (2006) Real-time PCR for detection of herpes simplex virus without nucleic acid extraction. BMC Infect Dis 6, 104

Perez-Sayas, C., T. Pina, M.A. Gomez-Martinez, G. Camanes, M.V. Ibanez-Gual, J.A. Jaques, M.A. Hurtado (2015) Disentangling mite predator-prey relationships by multiplex PCR. Mol Ecol Res 15, 1330-1345 doi:10.1111/1755-0998.12409

Perrot-Minnot, M.J., B. Cheval, A. Migeon, M. Navajas (2002) Contrasting effects of Wolbachia on cytoplasmic incompatibility and fecundity in the haplodiploid mite Tetranychus urticae. J Evol Biol 15, 808-817 doi:10.1046/j.1420-9101.2002.00446.x 
Ros, V.I.D., J.A.J. Breeuwer (2009) The effects of, and interactions between, Cardinium and Wolbachia in the doubly infected spider mite Bryobia sarothamni. Heredity 102, 413422 doi:10.1038/hdy.2009.4

Ros, V.I.D., V.M. Fleming, E.J. Feil, J.A.J. Breeuwer (2012) Diversity and recombination in Wolbachia and Cardinium from Bryobia spider mites. BMC Microbiol 12, S13

Sarmento, R.A., F. Lemos, C.R. Dias, W.T. Kikuchi, J.C.P. Rodrigues, A. Pallini, M.W. Sabelis, A. Janssen (2011) A herbivorous mite down-regulates plant defence and produces web to exclude competitors. Plos One 6, e23757 doi:10.1371/journal.pone.0023757

Sato, Y., J.M. Alba, M.W. Sabelis (2014) Testing for reproductive interference in the population dynamics of two congeneric species of herbivorous mites. Heredity 113, 495-502 doi:10.1038/hdy.2014.53

Shim, J.K., T.M. Khaing, H.E. Seo, J.Y. Ahn, D.O. Jung, J.H. Lee, K.Y. Lee (2016) Development of species-specific primers for rapid diagnosis of Tetranychus urticae,

Simões, P.M., G. Mialdea, D. Reiss, M.F. Sagot, S. Charlat (2011) Wolbachia detection: an T. kanzawai, T. phaselus and T. truncatus (Acari: Tetranychidae). Entomol Res 46, assessment of standard PCR Protocols. Mol Ecol Res 11, 567-572 doi:10.1111/j.17550998.2010.02955.x

Sint, D., L. Raso, M. Traugott (2012) Advances in multiplex PCR: balancing primer efficiencies and improving detection success. Methods Ecol Evol 3, 898-905 doi:10.1111/j.2041-210X.2012.00215.x 
505 Skoracka, A., S. Magalhães, B.G. Rector, L. Kuczynski (2015) Cryptic speciation in the

506

507

508

509

510

511

512

513

514 Acari: a function of species lifestyles or our ability to separate species? Exp Appl Acarol 67, 165-182 doi:10.1007/s10493-015-9954-8

Staudacher, H., B.C.J. Schimmel, M.M. Lamers, N. Wybouw, A.T. Groot, M.R. Kant (2017) Independent effects of a herbivore's bacterial symbionts on its performance and induced plant defences. Int J Mol Sci 18, 182 doi:10.3390/ijms18010182

Staudacher, K., P. Pitterl, L. Furlan, P.C. Cate, M. Traugott (2011) PCR-based species identification of Agriotes larvae. Bull Entomol Res 101, 201-210 doi: $10.1017 / \mathrm{s} 0007485310000337$

Suh, E., C. Sim, J.-J. Park, K. Cho (2015) Inter-population variation for Wolbachia induced reproductive incompatibility in the haplodiploid mite Tetranychus urticae. Exp Appl Acarol 65, 55-71 doi:10.1007/s10493-014-9846-3

Vala, F., J.A.J. Breeuwer, M.W. Sabelis (2000) Wolbachia-induced 'hybrid breakdown' in the two-spotted spider mite Tetranychus urticae Koch. Proc Roy Soc B-Biol Sci 267, $1931-1937$

Vala, F., A. Weeks, D. Claessen, J.A.J. Breeuwer, M.W. Sabelis (2002) Within- and betweenpopulation variation for Wolbachia-induced reproductive incompatibility in a haplodiploid mite. Evolution 56, 1331-1339

Weeks, A.R., J.A.J. Breeuwer (2001) Wolbachia-induced parthenogenesis in a genus of phytophagous mites. Proc Roy Soc Lond B Bio 268, 2245-2251 doi:10.1098/rspb.2001.1797

Weeks, A.R., F. Marec, J.A.J. Breeuwer (2001) A mite species that consists entirely of haploid females. Science 292, 2479-2482 doi:10.1126/science.1060411 
528 Weinert, L.A., E.V. Araujo-Jnr, M.Z. Ahmed, J.J. Welch (2015) The incidence of bacterial endosymbionts in terrestrial arthropods. Proc Roy Soc London 282, 20150249 doi:10.1098/rspb.2015.0249

531 Werren, J.H., L. Baldo, M.E. Clark (2008) Wolbachia: master manipulators of invertebrate biology. Nat Rev Microbiol 6, 741-751 doi:10.1038/nrmicro1969

533 Xi, Z.Y., C.C.H. Khoo, S.L. Dobson (2005) Wolbachia establishment and invasion in an Aedes aegypti laboratory population. Science 310, 326-328 doi:10.1126/science. 1117607

Xie, R.R., X.L. Chen, X.Y. Hong (2011) Variable fitness and reproductive effects of Wolbachia infection in populations of the two-spotted spider mite Tetranychus urticae Koch in China. Appl Entomol Zool 46, 95-102 doi:10.1007/s13355-010-0014-x

Xie, R.R., J.T. Sun, X.F. Xue, X.Y. Hong (2016) Cytoplasmic incompatibility and fitness benefits in the two-spotted spider mite Tetranychus urticae (red form) doubly infected

Yu, M.Z., K.J. Zhang, X.F. Xue, X.Y. Hong (2011) Effects of Wolbachia on mtDNA with Wolbachia and Cardinium. Syst Appl Acarol 21, 1161-1173 doi:10.11158/saa.21.9.1

\section{6} variation and evolution in natural populations of Tetranychus urticae Koch. Insect Mol Biol 20, 311-321 doi:10.1111/j.1365-2583.2010.01066.x

Zeh, J.A., M.M. Bonilla, A.J. Adrian, S. Mesfin, D.W. Zeh (2012) From father to son: transgenerational effect of tetracycline on sperm viability. Sci Rep 2, 375 doi:10.1038/srep00375

Zélé, F., I. Santos, I. Olivieri, M. Weill, O. Duron, S. Magalhães (2018) Endosymbiont diversity and prevalence in herbivorous spider mite populations in South-Western Europe. FEMS Microbiology Ecology in press doi:10.1093/femsec/fiy015 
Zélé, F., J. Vézilier, G. L'Ambert, A. Nicot, S. Gandon, A. Rivero, O. Duron (2014) Dynamics of prevalence and diversity of avian malaria infections in wild Culex pipiens mosquitoes: the effects of Wolbachia, filarial nematodes and insecticide resistance. Parasite Vector 7, 437 doi:10.1186/1756-3305-7-437

Zhang, Y.K., Y.T. Chen, K. Yang, G.X. Qiao, X.Y. Hong (2016) Screening of spider mites (Acari: Tetranychidae) for reproductive endosymbionts reveals links between coinfection and evolutionary history. Sci Rep 6, 27900 doi:10.1038/srep27900

Zhao, D.X., D.S. Chen, C. Ge, T. Gotoh, X.Y. Hong (2013a) Multiple Infections with Cardinium and two strains of Wolbachia in the spider mite Tetranychus phaselus Ehara: Revealing new forces driving the spread of Wolbachia. Plos One 8, e54964 doi:10.1371/journal.pone.0054964

Zhao, D.X., X.F. Zhang, X.Y. Hong (2013b) Host-symbiont interactions in spider mite Tetranychus truncates doubly infected with Wolbachia and Cardinium. Environ Entomol 42, 445-452 doi:10.1603/en12354

Zhu, L.Y., K.J. Zhang, Y.K. Zhang, C. Ge, T. Gotoh, X.Y. Hong (2012) Wolbachia strengthens Cardinium-induced cytoplasmic incompatibility in the spider mite Tetranychus piercei McGregor. Curr Microbiol 65, 516-523 doi:10.1007/s00284-0120190-8 
Table 1. List of primers used in multiplex to identify Tetranychus urticae, T. ludeni and T. evansi. We used a Tetranychus-generalist forward primer but three different speciesspecific reverse primers. "Concentration" corresponds to the concentration of each primer in the final mix.

\begin{tabular}{|c|c|c|c|c|}
\hline Target gene & Primer name & Sequence (5'->3') & Concentration & Product size \\
\hline Tetranychus 5.8S & TspG_F & TAATCGGTGCGAATTGCAGG & $2 \mu \mathrm{M}$ & - \\
\hline T. urticae $28 \mathrm{~S}$ & TuS_R & ATGTTTATTTGTGTTGTTTGCAAGC & $2 \mu \mathrm{M}$ & $550-\mathrm{bp}$ \\
\hline T. ludeni ITS2 & TeS_R & GAATGAAATAGATACTATTTGTGATTC & $3 \mu \mathrm{M}$ & 454-bp \\
\hline T. evansi ITS2 & TIS_R & GATTCATGTATACAYATATAAATATATGC & $3 \mu \mathrm{M}$ & 189-bp \\
\hline
\end{tabular}

Table 2. Reaction set-up using QIAGEN Multiplex PCR materials. The volume per reaction of each component is given for PCRs using either DNA extracts or entire mites.

\begin{tabular}{lll}
\hline Component & DNA extract & 1 mite \\
\hline 2x QIAGEN Multiplex PCR MasterMix & $5 \mu \mathrm{L}$ & $5 \mu \mathrm{L}$ \\
Primers mix & $2 \mu \mathrm{L}$ & $2 \mu \mathrm{L}$ \\
Q-solution & $2 \mu \mathrm{L}$ & $1 \mu \mathrm{L}$ \\
DEPC-water & - & $2 \mu \mathrm{L}$ \\
DNA & $1 \mu \mathrm{L}$ & - \\
\hline
\end{tabular}

Table 3. List of primers used in multiplex for simultaneous detection of Wolbachia, Cardinium and Rickettsia infections. Spider-mite generalist primers were used to control for DNA quality, thus to distinguish uninfected individuals from PCR failure. "Concentration" corresponds to the concentration of each primer in the final mix.

\begin{tabular}{|c|c|c|c|c|}
\hline Target gene & Primer name & Sequence (5'->3') & Concentration & Product size \\
\hline Spider mite partial & ITS1G_F & AGGTGAACCTGCGGAAGGATCATTAACG & $0.5 \mu \mathrm{M}$ & \multirow{2}{*}{$365-385-b p$} \\
\hline ITS1 & ITS1G_R & СCTTCTTTAAACCTTGCCGTCAGCATAAGC & $0.5 \mu \mathrm{M}$ & \\
\hline \multirow{2}{*}{$\begin{array}{l}\text { Rickettsia } \\
\text { gtlA }\end{array}$} & RICTG F & AGGCTAATGGGCTTTGGTCATCGTGTAT & $2 \mu \mathrm{M}$ & \multirow[b]{2}{*}{ 293-bp } \\
\hline & RICTG_R & TGTGCCATCCAGCCTACTGTTCTTGC & $2 \mu \mathrm{M}$ & \\
\hline \multirow{2}{*}{$\begin{array}{l}\text { Wolbachia } \\
\text { wsp }\end{array}$} & WSPTG_F & GTTGGTGTTGGTGCAGCGTATGTAAGC & $2 \mu \mathrm{M}$ & \multirow{2}{*}{ 222-bp } \\
\hline & WSPTG_R & AGTGCTGTAAAGAACTTTGATTCCGCCATC & $2 \mu \mathrm{M}$ & \\
\hline \multirow{2}{*}{$\begin{array}{l}\text { Cardinium } \\
16 \mathrm{~S} \text { rRNA }\end{array}$} & CARDTG_F & GGCGGCTTATTAAGTCAGTTGTGAAATCCT & $3 \mu \mathrm{M}$ & \multirow{2}{*}{$152-b p$} \\
\hline & CARDTG_R & GCTGCCTACGCTATTGGTATTCCTTATGAT & $3 \mu \mathrm{M}$ & \\
\hline
\end{tabular}

This is a post-peer-review, precopyedit version of an article published in Experimental and Applied Acarology. The final authenticated version is available online at:

https://doi.org/10.1007/s10493-018-0224-4 


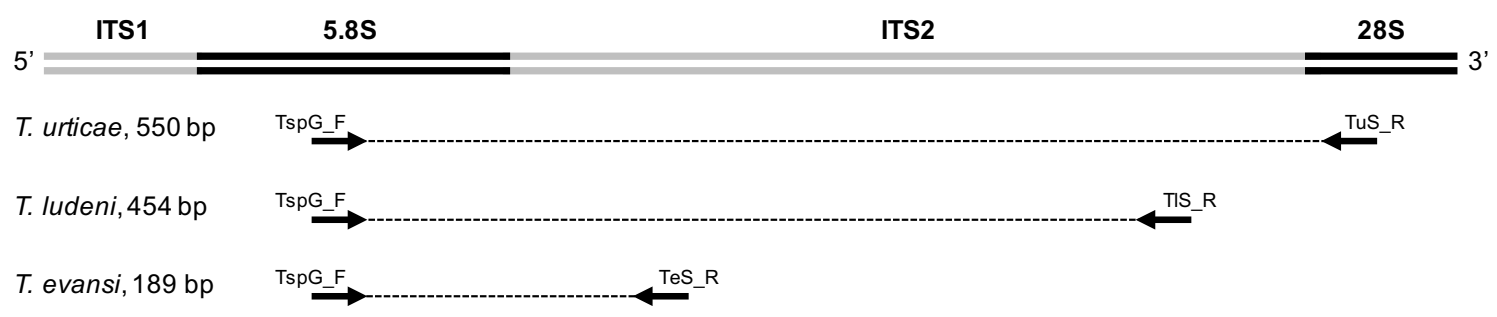

Fig. 1 Schematic overview of Tetranychus-generalist and species-specific primers targeting the partial 5.8S rDNA, ITS2 and $28 \mathrm{~S}$ gene sequences (full primer sequences are listed in Table 1 and Table 3), and corresponding PCR products allowing the identification of $T$. urticae, T. ludeni and $T$. evansi in a multiplex PCR. '_F' and '_R' denote forward and reverse primers, respectively.

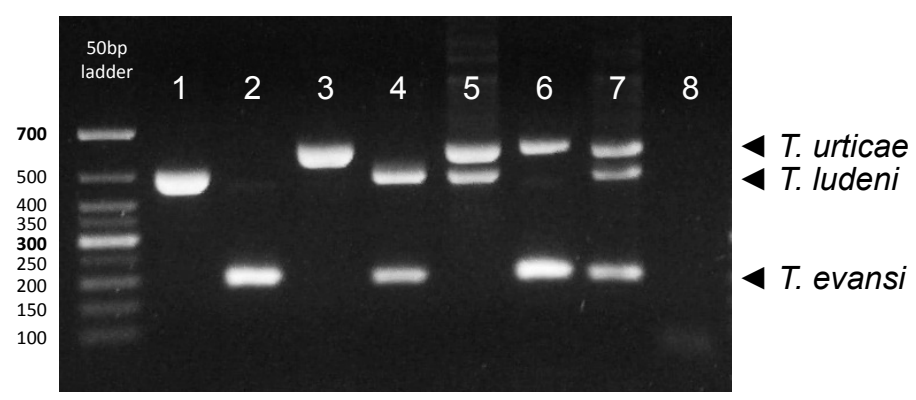

Fig. 2 Amplification profile of the multiplex PCR allowing the identification of $T$. urticae, T. Iudeni and T. evansi. The size fragment of the spider-mite partial 5.8S rDNA, ITS2 and 28S rDNA rDNA amplified by each specific primer pair is given in Table 1 . The position of amplicons generated from each spider-mite species are shown on the right part of the picture. Lane 1: T. ludeni; Lane 2: T. evansi; Lane 3: T. urticae, Lane 4: co-occurrence $T$. ludeni -T. evansi; Lane 5: co-occurrence T. urticae - T. ludeni; Lane 6: co-occurrence $T$. urticae - T. evansi; Lane 7: co-occurrence of the three species; Lane 8: negative control without DNA template. 


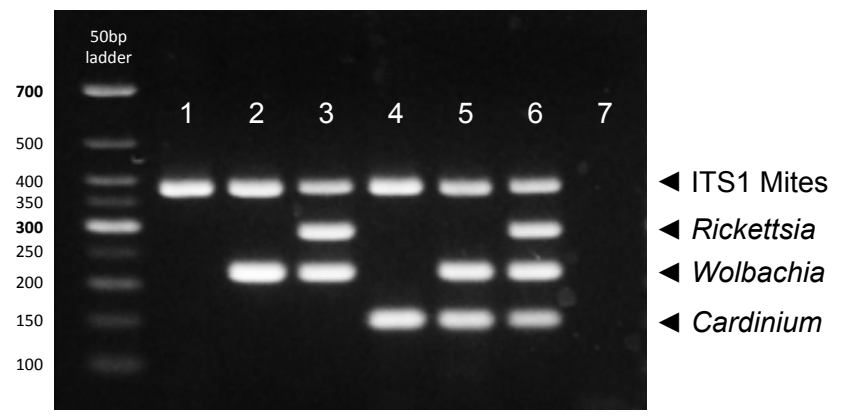

Fig. 3 Amplification profile of the multiplex PCR allowing the simultaneous detection of Wolbachia, Cardinium and Rickettsia in spider mites. The size of the amplified fragment for each specific primer pair is given in Table 3. The position of amplicons generated from each symbiont are shown on the right part of the picture. DNA quality was controlled by amplifying a fragment of the spider-mite partial ITS1 rDNA. The presence of several bands indicates coinfections. Lane 1: uninfected mite; Lane 2: Wolbachia single infection; Lane 3: Rickettsia-Wolbachia coinfection, Lane 4: Cardinium single infection; Lane 5: WolbachiaCardinium coinfection; Lane 6: triple infection; Lane 7: negative control without DNA template.

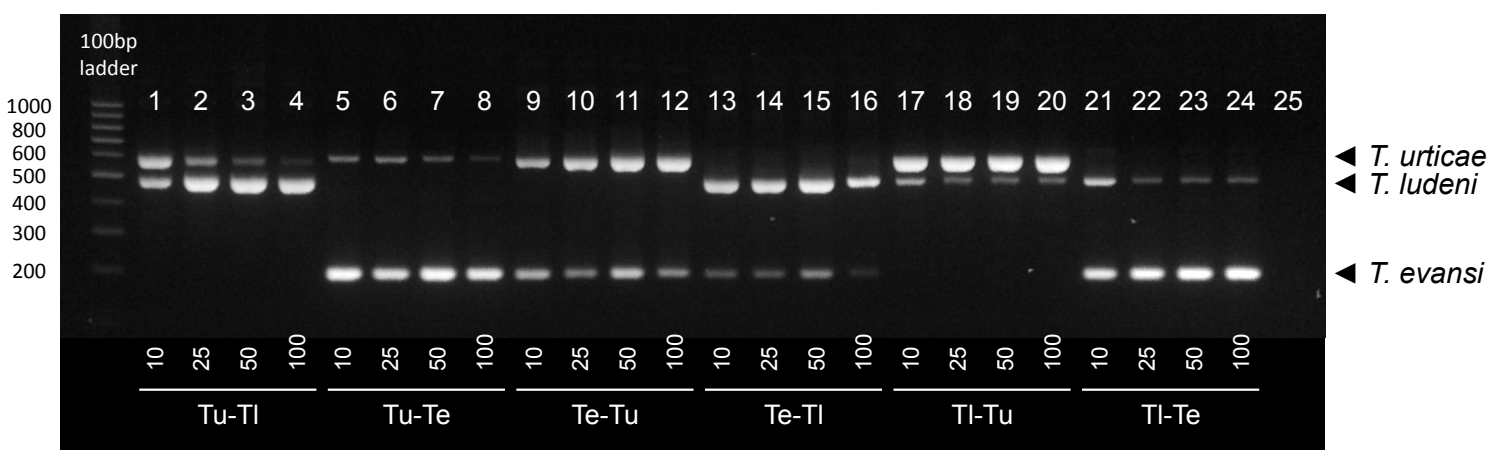

Fig. 4 Multiplex sensitivity to detect contaminations by mites from a different species in different pool sizes. The position of amplicons generated from each spider-mite species are shown on the right part of the picture. Pool sizes (10, 25, 50 and 100 females) and compositions are given under the lanes. Tu-Tl (lanes 1-4): one T. urticae (Tu) female in pools of T. ludeni (Tl) females; Tu-Te (lanes 5-8): one Tu female in pools of T. evansi (Te) females; Te-Tu (lanes 9-12): one Te female in pools of Tu females; Te-Tl (lanes 13-16): one Te female in pools of $\mathrm{Tl}$ females; $\mathrm{Tl}-\mathrm{Tu}$ (lanes 17-20): one $\mathrm{Tl}$ female in pools of $\mathrm{Tu}$ females; $\mathrm{Tl}-\mathrm{Te}$ (lanes 21-24): one Tl female in pools of Te females; Negative control: no DNA template (lane $25)$. 


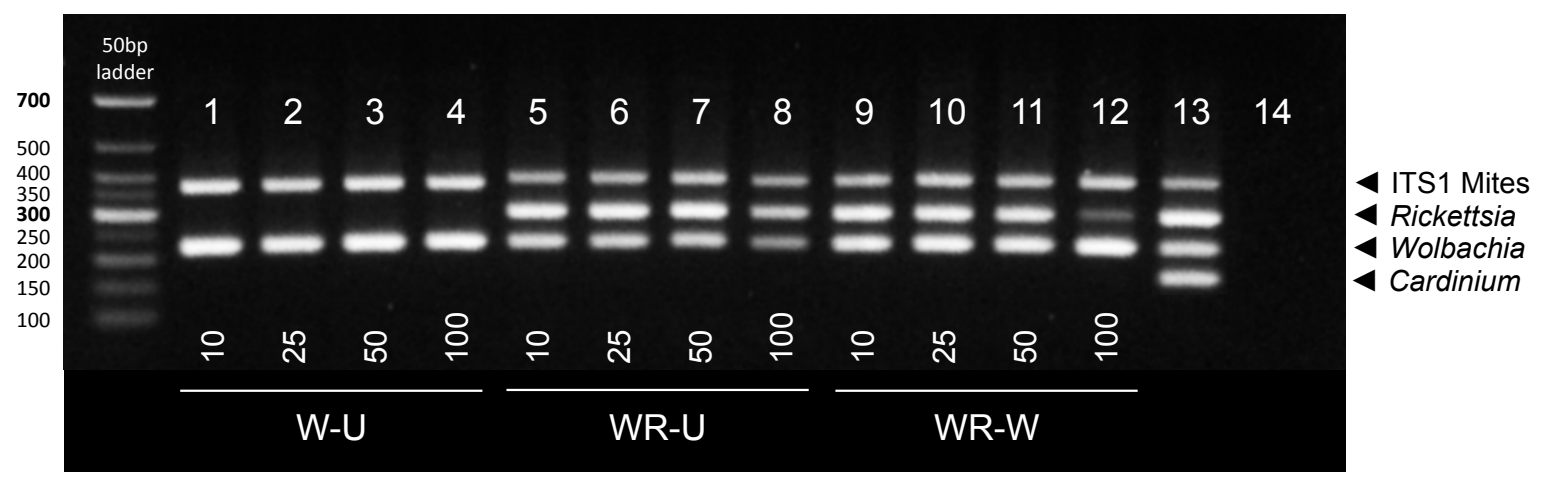

Fig. 5 Multiplex sensitivity to detect contaminations by mites with different infection types within different pool sizes. The position of amplicons generated from each symbiont, along with a fragment of the spider-mite ITS1 rDNA, are shown on the right part of the picture. Pool sizes $(10,25,50$ and 100 females) and compositions are given under the lanes. W-U (lanes 1-4): one Wolbachia-infected (W) female in pools of uninfected (U) females; WR-U (lanes 5-8): one Wolbachia-Rickettsia coinfected (WR) female in pools of U females; WR-W (lanes 9-12): one WR female in pools of W females; Positive control: DNA template with Rickettsia-Wolbachia-Cardinium coinfection (lane 13); Negative control: no DNA template (lane 14).

(a)

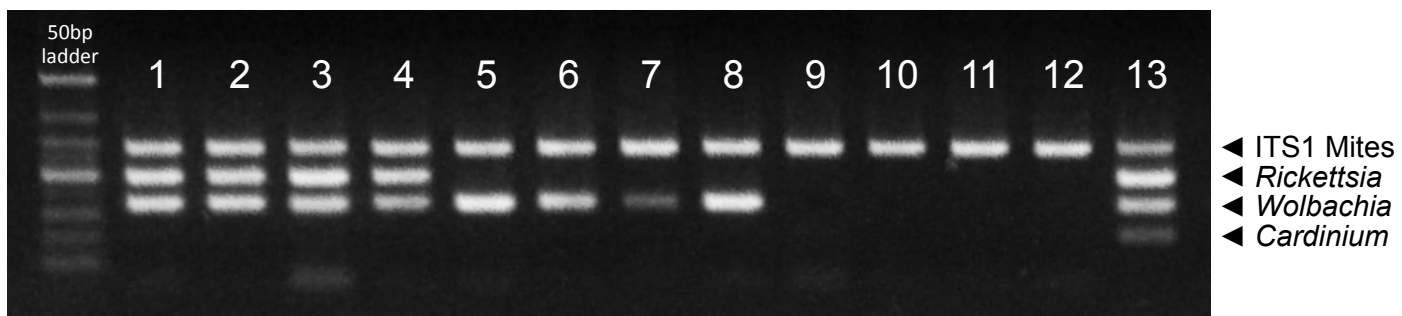

(b)

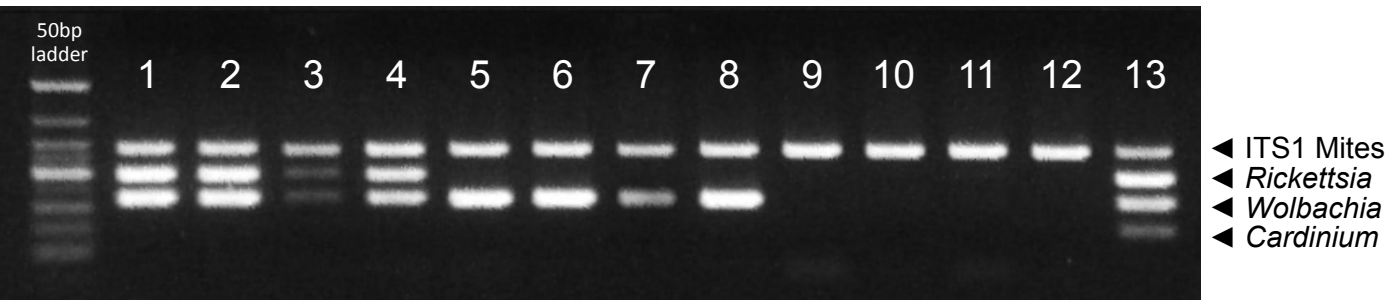

Fig. 6 Simultaneous detection of Wolbachia, Cardinium and Rickettsia in non-extracted DNA from adult females (a) and males (b) of $\boldsymbol{T}$. urticae. The position of amplicons generated from each symbiont, along with a fragment of the spider-mite ITS1 rDNA, are shown on the right part of the picture. Lane 1-4, Rickettsia-Wolbachia coinfection; Lane 5-8, Wolbachia single infection; Lane 9-12, uninfected mite, Lane 13, positive control (DNA template with Rickettsia-Wolbachia-Cardinium coinfection). 
(a)

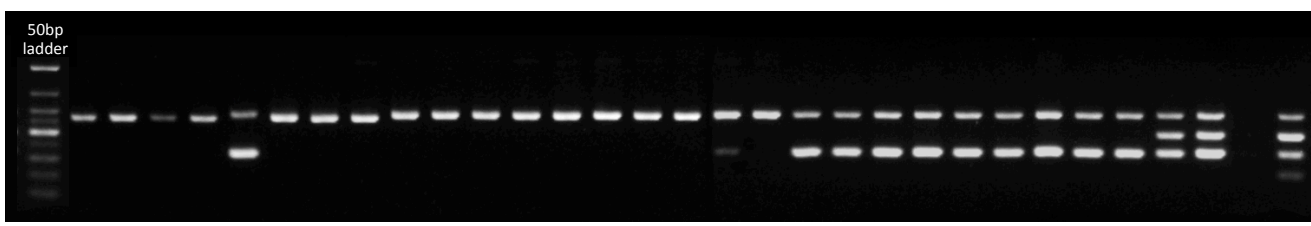

(b)

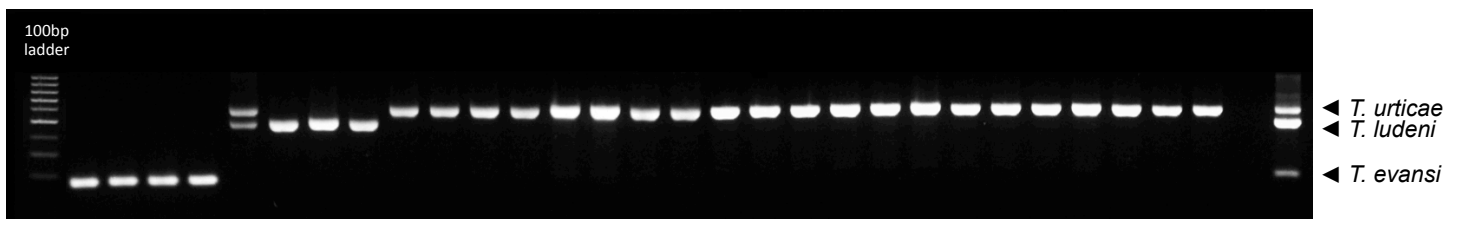

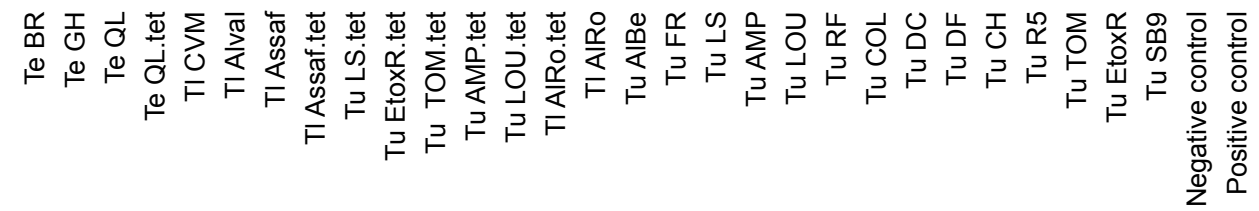

Fig. 7 Application of both multiplex PCRs to detect contamination within laboratory populations of Tetranychus mites. (a) symbiont infection status, and (b) species identification for 29 different populations of T. evansi $(\mathrm{Te} ; \mathrm{n}=4)$, T. ludeni $(\mathrm{Tl} ; \mathrm{n}=4)$, or $T$. urticae $(\mathrm{Tu} ; \mathrm{n}=21)$. The name of each laboratory population is on the bottom of the picture. The position of amplicons generated from each symbiont, along with a fragment of the spidermite ITS2 gene (a), or from each spider-mite species (b) are shown on the right part of the picture. The infection status of each population was known prior to the PCR, only 9 populations of T. urticae being infected by Wolbachia (from Tu AMP to Tu TOM), and 2 coinfected by Wolbachia and Rickettsia (Tu EtoxR and Tu SB9). 